Research paper

\title{
Blood neutrophils from children with COVID-19 exhibit both inflammatory and anti-inflammatory markers
}

\author{
Vanesa Seery $^{\mathrm{a}}$, Silvina C. Raiden ${ }^{\mathrm{b}}$, Silvia C. Algieric ${ }^{\mathrm{c}}$, Nicolás A. Grisolía ${ }^{\mathrm{b}}$, Daniela Filippo ${ }^{\mathrm{d}}$, \\ Norberto De Carli ${ }^{\mathrm{e}}$, Sandra Di Lalla ${ }^{\mathrm{f}}$, Héctor Cairoli ${ }^{\mathrm{b}}$, María J. Chiolo ${ }^{\mathrm{g}}$, Claudia N. Meregalli ${ }^{\mathrm{h}}$, \\ Lorena I. Gimenez ${ }^{\mathrm{d}}$, Gabriela Gregorio ${ }^{c}$, Mariam Sarlii, Ana L. Alcalde ${ }^{\mathrm{c}}$, Carolina Davenport ${ }^{\mathrm{b}}$, \\ María J. Bruera ${ }^{i}$, Nancy Simaz ${ }^{c}$, Mariela F. Pérez ${ }^{c}$, Valeria Nivela ${ }^{j}$, Carola Bayle ${ }^{j}$, \\ Patricia Tuccillok ${ }^{k}$, María T. Agostak ${ }^{\mathrm{k}}$, Hernán Pérez ${ }^{\mathrm{k}, 1}$, Susana Villa Noval', Patricia Suárez', \\ Eugenia M. Takata ${ }^{1}$, Mariela García ${ }^{1,1}$, Jorge Lattner ${ }^{\mathrm{m}}$, María J. Rolón ${ }^{\mathrm{n}}$, Patricia Coll ${ }^{\mathrm{n}}$, \\ Inés Sananez ${ }^{\mathrm{a}}$, María P. Holgado ${ }^{\mathrm{a}}$, Fernando Ferrero ${ }^{\mathrm{b}}$, Jorge Geffner ${ }^{\mathrm{a}}$, Lourdes Arruvito ${ }^{\mathrm{a}, *}, 1$ in \\ behalf of the COVID-19 Naval Pediatric Workgroup and in behalf of the COVID-19, Fernández \\ Pediatric Residency Workgroup
}

\footnotetext{
a Instituto de Investigaciones Biomédicas en Retrovirus y SIDA, Facultad de Medicina. UBA-CONICET, Paraguay 2155, C1121ABG CABA, Argentina

b Departamento de Medicina, Hospital General de Niños Pedro de Elizalde. Av. Montes de Oca 40, CABA C1270, Argentina

' Servicio de Pediatría, Hospital Nacional Profesor Alejandro Posadas, Marconi Morón 386, Buenos Aires B1684, Argentina

${ }^{\mathrm{d}}$ Servicio de Pediatría, Hospital Municipal Diego Thompson. Avellaneda 33, Buenos Aires B1650, Argentina

e Servicio de Pediatría, Clínica del Niño de Ouilmes, Av. Lamadrid 444, Buenos Aires B1878, Argentina

${ }^{\mathrm{f}}$ Departamento de Consultorios Externos, Hospital General de Niños Pedro de Elizalde, Av. Montes de Oca 40, CABA C1270, Argentina

${ }^{\mathrm{g}}$ Departamento de Cirugía, Hospital General de Niños Pedro de Elizalde, Av. Montes de Oca 40, CABA C1270, Argentina

${ }^{\mathrm{h}}$ Unidad de Terapia Intensiva Pediátrica, Departamento de Urgencias, Hospital General de Niños Pedro de Elizalde. Av. Montes de Oca 40, CABA C1270, Argentina

${ }^{\mathrm{i}}$ Unidad de Terapia Intensiva Pediátrica, Hospital Nacional Profesor Alejandro Posadas, Marconi Morón 386, Buenos Aires B1684, Argentina

j Departamento de Emergencias Pediátrica, Hospital Nacional Profesor Alejandro Posadas, Marconi Morón 386, Buenos Aires B1684, Argentina

${ }^{\mathrm{k}}$ Servicio de Pediatría, Hospital Naval Cirujano Mayor Dr. Pedro Mallo, Av. Patricias Argentinas 351, CABA C1405, Argentina

${ }^{1}$ Servicio de Pediatría, Hospital General de Agudos Dr. Juan A. Fernández, Av. Cerviño 3356, CABA C1425, Argentina

m Servicio de Infectología Pediátrica, Hospital Naval Cirujano Mayor Dr. Pedro Mallo, Av. Patricias Argentinas 351, CABA C1405, Argentina

n División Infectología, Hospital General de Agudos Dr. Juan A. Fernández, Av. Cerviño 3356, CABA C1425, Argentina
}

\section{A R T I C L E I N F O}

\section{Article History:}

Received 27 January 2021

Revised 10 March 2021

Accepted 12 April 2021

Available online 9 May 2021

\section{Keywords:}

Pediatric COVID-19

Neutrophils

Fc $\gamma$ receptor

Cell-adhesion molecules

Inhibitory Receptors

$\operatorname{IgG}$

\begin{abstract}
A B S T R A C T
Background: Perhaps reflecting that children with COVID-19 rarely exhibit severe respiratory symptoms and often remain asymptomatic, little attention has been paid to explore the immune response in pediatric COVID-19. Here, we analyzed the phenotype and function of circulating neutrophils from children with COVID-19.

Methods: An observational study including 182 children with COVID-19, 21 children with multisystem inflammatory syndrome (MIS-C), and 40 healthy children was performed in Buenos Aires, Argentina. Neutrophil phenotype was analyzed by flow cytometry in blood samples. Cytokine production, plasma levels of IgG antibodies directed to the spike protein of SARS-CoV-2 and citrullinated histone H3 were measured by ELISA. Cell-free DNA was quantified by fluorometry.

Findings: Compared with healthy controls, neutrophils from children with COVID-19 showed a lower expression of CD11b, CD66b, and L-selectin but a higher expression of the activation markers HLA-DR, CD64 and PECAM-1 and the inhibitory receptors LAIR-1 and PD-L1. No differences in the production of cytokines and NETs were observed. Interestingly, the expression of CD64 in neutrophils and the serum concentration of IgG antibodies directed to the spike protein of SARS-CoV-2 distinguished asymptomatic from mild and moderate COVID-19.

Interpretation: Acute lung injury is a prominent feature of severe COVID-19 in adults. A low expression of adhesion molecules together with a high expression of inhibitory receptors in neutrophils from children with COVID-19 might prevent tissue infiltration by neutrophils preserving lung function.
\end{abstract}

\footnotetext{
* Corresponding author: Instituto de Investigaciones Biomédicas en Retrovirus y SIDA. Facultad de Medicina. UBA-CONICET, Paraguay 2155, C1121ABG CABA, Argentina. E-mail address: arruvitol@gmail.com (L. Arruvito).

1 Members are listed in supplementary appendix.
} 
Funding: This study was supported by the Ministry of Science and Technology (National Agency for Scientific and Technological Promotion, IP-COVID-19-0277 and PMO BID PICT 2018-2548), and University of Buenos Aires from Argentina (20020170100573BA).

(c) 2021 The Authors. Published by Elsevier B.V. This is an open access article under the CC BY-NC-ND license (http://creativecommons.org/licenses/by-nc-nd/4.0/)

\section{Research in context}

\section{Evidence before this study}

Severe Coronavirus induced disease 2019 (COVID-19) in children is rare being the clinical course of the infection typically mild and often asymptomatic. The reasons for this remain unclear. Very little is known about the immune response against SARS-CoV-2 in children and no previous reports have analyzed the phenotype and function of neutrophils in pediatric COVID-19.

\section{Added value of this study}

Neutrophils from children with COVID-19 express an atypical phenotype characterized by a low expression of adhesion molecules and a high expression of the inhibitory receptors LAIR-1 and PD-L1 and the activation markers HLA-DR, CD64, and PECAM-1. The expression of CD64 in neutrophils and the serum concentration of IgG antibodies directed to the spike protein of SARS-CoV-2 were higher in symptomatic patients compared with asymptomatic ones, and might represent useful biomarkers to stratify patients at admission.

\section{Implications of all the available evidence}

This study demonstrates the acquisition of an atypical phenotype by circulating neutrophils in children with COVID-19 that markedly differs from the phenotype showed by neutrophils from adult patients with COVID-19. We hypothesize that this particular signature might prevent tissue infiltration by neutrophils preserving lung function in children with COVID-19.

\section{Introduction}

Among the most intriguing observations in Coronavirus disease 2019 (COVID-19) is the reduced incidence and mortality in children [1]. Clinical manifestations usually include fever, cough, nasal congestion, sore throat and fatigue, and up to one-third of the infected children remains asymptomatic [2]. Epidemiological data from Argentina as of December 9, 2020, show that only $0.3 \%$ of infected children were admitted to Pediatric Intensive Care Unit (PICU) and less than $0.1 \%$ required mechanical ventilation (https://www.argen tina.gob.ar/salud/coronavirus-COVID-19/sala-situacion). The view that acute respiratory syndrome coronavirus 2 (SARS-CoV-2) infection in children is always associated to a mild disease has been challenged by the emergence of a novel multisystem inflammatory syndrome in children (MIS-C) associated to a previous infection by SARS-CoV-2 [3].

The reasons underlying the mild and often asymptomatic course of COVID-19 in children are unclear. A decreased expression of host factors required for the progression of viral infection in the airway epithelium such as the viral entry receptor angiotensin-converting enzyme 2 and the transmembrane serine protease 2 might explain, at least in part, the mild clinical course of pediatric infection [4-6]. On the other hand, differences in the immune response between children and adults might also be involved [7-9]. In recent months, several works directed to analyze the immune response in MIS-C have been published. However, not enough attention has been paid to study the immune response in children during the acute phase of COVID-19. The observations made in this field are very scarce and do not allow to establish a causal relationship between the immune response and the course of infection.

Here, we focus on the phenotype and function of neutrophils in pediatric COVID-19. We found that neutrophils from infected children express a particular signature characterized by a reduced expression of adhesion molecules involved in neutrophil migration together with an increased expression of both, the inflammatory markers CD64, HLA-DR and PECAM-1 and the inhibitory receptors leukocyte-associated immunoglobulin-like receptor 1 (LAIR-1) and programmed death-ligand 1 (PD-L1). Moreover, we found that symptomatic and asymptomatic children with COVID-19 can be differentiated based on the neutrophil expression of CD64 and the plasma levels of IgG antibodies against the spike protein of SARS-CoV-2.

\section{Methods}

\subsection{Study population}

This observational study was conducted in Buenos Aires City and Buenos Aires province, Argentina, between May and October 2020. We recruited girls, boys and adolescents aged between 5 months and 15 years admitted to the Hospital General de Niños Pedro de Elizalde, Hospital Nacional Prof. Alejandro Posadas, Hospital Municipal Diego Thompson, Clínica del Niño de Quilmes, Hospital Naval Cirujano Mayor Dr. Pedro Mallo and Hospital General de Agudos Dr. J. A Fernández. Patients were divided into two main cohorts: 1- patients with active SARS-CoV-2 infection confirmed by PCR of nasopharyngeal swabs (COVID-19, $n=174$ ) and, 2- MIS-C patients with previous SARS-CoV-2 infection confirmed by PCR of nasopharyngeal swabs and/or SARS-CoV-2 IgG antibody-positive serology $(n=21)$. Disease severity was classified into four clinical types (asymptomatic, mild, moderate and severe), according to the Health Ministry from Argentina and the WHO interim guidance. Asymptomatic patients $(n=29)$ remain asymptomatic throughout the course of the infection. They were admitted after SARS-CoV-2 infection was confirmed by PCR. Mild patients $(n=102)$ do not present evidence of viral pneumonia or hypoxia. Symptoms are non-specific and could include fever, fatigue, headache, myalgia, cough, abdominal pain and diarrhea. Moderate patients $(n=43)$ present symptoms and signs of nonsevere pneumonia (cough or fast breathing and/or chest indrawing), and include patients with comorbidities such as cardiac or respiratory disease, immunodeficiency and prematurity. Our cohort did not contain children under severe disease. Characteristics of children with COVID-19 are shown in Table 1 and Table S1. MIS-C was diagnosed according to WHO criteria: -fever during more than 3 days; -two of the following signs (rash or bilateral non-purulent conjunctivitis, mucocutaneous inflammation signs, hypotension, shock, myocardial dysfunction, pericarditis, valvulitis, coronary abnormalities, coagulopathy, and/or acute gastrointestinal symptoms); -elevated markers of inflammation, -no other microbial cause; and -evidence of previous SARS-CoV-2 infection (PCR or serology positive). Clinical features of MIS-C are shown in Table 1 and Table S2. Eight children who had recovered from COVID-19 disease (4 to 5 weeks post illness-onset) were also included. Data are shown in Table S3. Our control cohort included forty children age and sex matched that were admitted to 
Table 1

Characteristics of children with COVID-19, MIS-C and healthy controls

\begin{tabular}{|c|c|c|c|c|}
\hline Characteristics & Control $(\mathrm{n}=40)$ & COVID-19 (n=174) & MIS-C $(n=21)$ & $P$ \\
\hline Age, years & $5(1-9)$ & $4(0.5-10)$ & $6(3-11)$ & \\
\hline$<1, n(\%)$ & $9(23)$ & $46(26)$ & $2(10)$ & \\
\hline $1-5, n(\%)$ & $17(42)$ & $52(30)$ & $8(38)$ & \\
\hline $6-10, n(\%)$ & $8(20)$ & $33(19)$ & $6(29)$ & \\
\hline$>10, n(\%)$ & $6(15)$ & $43(25)$ & $5(24)$ & \\
\hline Female, $\mathrm{n}(\%)$ & $24(60)$ & $80(46)$ & $5(24)$ & ${ }^{*}$ Control vs MIS-C \\
\hline SARS-CoV-2 PCR positive, $\mathrm{n}(\%)$ & 0 & $174(100)$ & $10(48)$ & \\
\hline SARS-CoV-2 IgG antibody positive, $\mathrm{n}(\%)$ & 0 & $53(31)$ & $19(90)$ & \\
\hline SARS-CoV-2 IgM antibody positive, $\mathrm{n}(\%)$ & 0 & $68(39)$ & $10(48)$ & \\
\hline Close contact positive, $\mathrm{n}(\%)$ & 0 & $117(67)$ & $13(62)$ & \\
\hline \multicolumn{5}{|l|}{ Laboratory } \\
\hline WBC counts, $10^{3} / \mathrm{mm}^{3}$ & $\mathrm{~N} / \mathrm{A}$ & $\begin{array}{c}7,600 \\
(5,100-10,125)\end{array}$ & $\begin{array}{c}10,600 \\
(8,300-16,900)\end{array}$ & ${ }^{* *}$ COVID-19 vs MIS-C \\
\hline Neutrophils, \% & $\mathrm{N} / \mathrm{A}$ & $45(31-67)$ & $78(66-90)$ & $* * * *$ COVID-19 vs MIS-C \\
\hline Lymphocytes, \% & $\mathrm{N} / \mathrm{A}$ & $38(20-54)$ & $23(11-34)$ & ${ }^{* *}$ COVID-19 vs MIS-C \\
\hline Neutrophil-to-lymphocyte ratio & $\mathrm{N} / \mathrm{A}$ & $1(0.6-4)$ & $4(2-9)$ & ${ }^{* *}$ COVID-19 vs MIS-C \\
\hline \multicolumn{5}{|l|}{ Coinfections, $\mathrm{n}(\%)$} \\
\hline None & $40(100)$ & $166(95)$ & $21(100)$ & \\
\hline Bacterial & 0 & $8(5)$ & 0 & \\
\hline Viral & 0 & 0 & 0 & \\
\hline \multicolumn{5}{|l|}{ Comorbidities, n (\%) } \\
\hline None & $37(92)$ & $136(78)$ & $16(76)$ & \\
\hline Heart disease & 0 & $1(1)$ & 0 & \\
\hline Renal disease & 0 & $2(1)$ & 0 & \\
\hline Lung disease & 0 & $20(12)$ & $4(19)$ & \\
\hline Prematurity & 0 & $3(2)$ & 0 & \\
\hline Autoimmunity & 0 & $3(2)$ & 0 & \\
\hline Cancer & 0 & $4(2)$ & 0 & \\
\hline Obesity & $2(5)$ & $4(2)$ & $1(5)$ & \\
\hline Diabetes & $1(3)$ & $1(1)$ & 0 & \\
\hline \multicolumn{5}{|l|}{ Signs and symptoms, n (\%) } \\
\hline Fever & $\mathrm{N} / \mathrm{A}$ & $81(47)$ & $20(95)$ & *****COVID-19 vs MIS-C \\
\hline Cough & $\mathrm{N} / \mathrm{A}$ & $34(20)$ & $2(10)$ & \\
\hline Dyspnea & $\mathrm{N} / \mathrm{A}$ & $16(9)$ & $1(5)$ & \\
\hline Odynophagia & $\mathrm{N} / \mathrm{A}$ & $24(14)$ & $4(19)$ & \\
\hline Myalgia & $\mathrm{N} / \mathrm{A}$ & $9(5)$ & $2(10)$ & \\
\hline Pneumonia & $\mathrm{N} / \mathrm{A}$ & $12(7)$ & $3(14)$ & \\
\hline Gastrointestinal & $\mathrm{N} / \mathrm{A}$ & $23(13)$ & $12(57)$ & ${ }^{* * * * *}$ COVID-19 vs MIS-C \\
\hline Haematological & $\mathrm{N} / \mathrm{A}$ & 0 & $12(57)$ & $* * * *$ COVID-19 vs MIS-C \\
\hline Mucocutaneous & $\mathrm{N} / \mathrm{A}$ & 0 & $8(38)$ & *****COVID-19 vs MIS-C \\
\hline Cardiac abnormalities & $\mathrm{N} / \mathrm{A}$ & 0 & $12(57)$ & *****COVID-19 vs MIS-C \\
\hline Neurological & $\mathrm{N} / \mathrm{A}$ & 0 & $1(5)$ & \\
\hline Shock & $\mathrm{N} / \mathrm{A}$ & 0 & $5(24)$ & *****COVID-19 vs MIS-C \\
\hline PICU admission, n (\%) & 0 & $2(1)$ & $12(57)$ & *****COVID-19 vs MIS-C \\
\hline \multicolumn{5}{|l|}{ Medications, $\mathrm{n}(\%)$} \\
\hline None & $40(100)$ & $148(85)$ & 0 & \\
\hline Corticosteroid & 0 & $18(10)$ & $20(95)$ & *****COVID-19 vs MIS-C \\
\hline IVIG & 0 & 0 & $20(95)$ & *****COVID-19 vs MIS-C \\
\hline Antibiotic & 0 & $8(5)$ & $2(10)$ & \\
\hline Enoxaparin & 0 & 0 & $14(67)$ & *****COVID-19 vs MIS-C \\
\hline Aspirin & 0 & 0 & $15(71)$ & *****COVID-19 vs MIS-C \\
\hline Inotropic & 0 & 0 & $9(43)$ & *****COVID-19 vs MIS-C \\
\hline \multicolumn{5}{|l|}{ Respiratory status, n(\%) } \\
\hline Mechanical ventilation & 0 & 0 & $5(24)$ & *****COVID-19 vs MIS-C \\
\hline Oxygen requirement & 0 & $28(16)$ & $1(5)$ & \\
\hline Room air & $40(100)$ & $146(84)$ & $16(76)$ & \\
\hline
\end{tabular}

Data are expressed as median values (25th-75th percentile) unless otherwise indicated. Abbreviations: IVIG, Intravenous Immunoglobulin; PICU, pediatric intensive care unit; WBCs, white blood cells. Mann-Whitney and Kruskall-Wallis tests were used for cuantitative variables. Fisher's exact test and Chi-square test were adopted for category variables. Only statistical differences are shown. ${ }^{*} \mathrm{p}<0.05,{ }^{* *} \mathrm{p}<0.01,{ }^{* * * *} \mathrm{p}<0.0001$.

the hospitals for routine screening and/or scheduled surgery. They had no history of recent respiratory infection or close-contact and they were negative for IgM and IgG antibodies directed to SARS-CoV-2.

\subsection{Study approval}

This study was conducted in accordance with the Declaration of Helsinki. The Institutional Review Board at institutions participants reviewed and approved the sample collection and the overall study
(Hospital General de Niños Pedro de Elizalde protocol reference 1226/20, and Hospital General de Agudos Dr. J. A Fernández protocol reference $1720 / 20$ ).

\subsection{Ethics statement}

Parents or legal guardians from children under 8 years provided written, informed consent. Children older than 8 years old provided written, informed assent and their parents or legal guardians also provided written, informed consent. 


\subsection{Blood samples}

$0.5-1 \mathrm{~mL}$ of whole blood samples were obtained within $72 \mathrm{~h}$ of hospital admission. After being centrifuged for $10 \mathrm{~min}$ at $1000 \mathrm{rpm}$, plasma was separated and stored at $-80{ }^{\circ} \mathrm{C}$ until used. Whole blood samples were used for flow cytometry and/or cell isolation.

\subsection{Neutrophil isolation}

Neutrophils were isolated within the first $3 \mathrm{~h}$ of blood collection by Ficoll-Hypaque Plus (GE Healthcare, 17144003) gradient centrifugation and dextran sedimentation (Sigma Aldrich, 31392), as described [10]. Cell suspensions containing $>96 \%$ neutrophils were suspended in RPMI 1640 medium (Sigma-Aldrich, R8758) with 10\% FCS (Natocor; complete medium).

\subsection{Immunophenotyping by flow cytometry}

Whole blood $(100 \mu \mathrm{L})$ was stained with the antibody cocktail mix for $20 \mathrm{~min}$ at room temperature. After being washed with PBS $0.1 \%$ BSA, erythrocytes were lysed and cells were fixed using Lysing buffer (BD Biosciences, 555899). The following conjugated-monoclonal antibodies were used: anti-CD14 (BV421, 367144), anti-CD15 (PerCP, 301922), anti-CD11b (PE-Cy7, 301322), anti-CD66b (FITC, 305104), anti-CD62L (L-selectin, PE, 304806), anti-HLA-DR (APC, 307610), anti-PECAM-1 (Pacific Blue, 303114), anti-LAIR-1 (Alexa Fluor 647, 342802), anti-PD-L1 (BV510, 329734), anti-CD16 (APC, 980104), antiCD32 (PE-Cy7, 303214) and anti-CD64 (FITC, 305006) all from Biolegend. Control samples were incubated with an isotype-matched antibody. Statistical analyses were based on at least 100,000 events gated on the population of interest. Data were acquired using a FACSCanto II (Becton Dickinson) and analyzed with FlowJov10.6.2.

\subsection{Neutrophil cultures}

To test the spontaneous cytokine secretion, neutrophils $\left(5 \times 10^{6} /\right.$ $\mathrm{mL}$ ) were suspended in complete medium and cultured in U-bottom 96-well plates (Greiner-Bio One, 650161) for $18 \mathrm{~h}$ at $37^{\circ} \mathrm{C}$. To test the response to TLR 7/8 agonist, neutrophils were stimulated with $5 \mu \mathrm{M}$ of resiquimod (R848, Sigma-Aldrich, SML0196). To test neutrophil extracellular traps (NETs) release, neutrophils $\left(1 \times 10^{6} / \mathrm{mL}\right)$ were suspended in FCS-free culture medium and cultured in 48-well plates (Greiner-Bio One, 677180) for $4 \mathrm{~h}$ at $37^{\circ} \mathrm{C}$. Then, cells were treated with a low concentration of DNase I ( $2 \mathrm{U} / \mathrm{mL}$; Promega, M6101) for 15 min at $37{ }^{\circ} \mathrm{C}$ to detach NET that remained attached to the neutrophil surface [11]. After the addition of EDTA ( $5 \mathrm{mM}$ ) to prevent further DNase activity [12], the samples were centrifuged and the supernatants were collected and store at $-80^{\circ} \mathrm{C}$ until use.

\subsection{Analysis of cytokine production}

Levels of IL-1 $\beta$ (437015), IL-6 (430501) and IL-8 (431501, Biolegend) in plasma and neutrophil supernatants were analyzed by ELISA according to the manufacturer's instructions.

\subsection{Cell-free DNA quantitation}

Cell-free DNA was quantified in plasma and/or in the supernatants of neutrophil cultures using the Quant-iT PicoGreen dsDNA Assay Kit (Molecular Probes, P11496) according to the manufacturer's instructions. Briefly, samples were diluted $1: 10$ or $1: 5$, for plasma and supernatants respectively, in $10 \mathrm{mM}$ Tris- $\mathrm{HCl} 1 \mathrm{mM}$ EDTA buffer, and Quant-iT PicoGreen dsDNA reagent was added in a 1:1 ratio. After incubation for $3 \mathrm{~min}$ at room temperature in the dark, the fluorescence was read in a SpectraMax i3x Multi-Mode Plate Reader
(Molecular Devices; ex. 480 nm, em. 520 nm). A standard curve was plotted and a regression analysis was applied.

\subsection{Quantification of citrullinated histone $H 3$}

Citrullinated histone $\mathrm{H} 3$ was quantified in plasma using the Citrullinated Histone H3 (Clone 11D3) ELISA Kit (Cayman, 501620) according to the manufacturer's instructions.

\subsection{Analysis of plasma SARS-CoV-2-specific IgG antibodies}

Plasma levels of IgG antibodies directed to the spike protein of SARSCoV-2 were analyzed by ELISA (COVIDAR IgG), as described [13]. Briefly, samples were diluted 1:10 in PBS-T containing 0.8\% casein and incubated for $1 \mathrm{~h}$ at $37^{\circ} \mathrm{C}$. After washing with PBS-T, HRP-conjugated goat mouse anti-human IgG antibodies was added and incubated for $30 \mathrm{~min}$ at $37{ }^{\circ} \mathrm{C}$, followed by TMB Substrate Reagent. The absorbance (OD) was measured at $450 \mathrm{~nm}$. The cut-off was calculated as the mean OD of the negative sera plus 0.2 . Samples with OD lower than the cut-off were considered negative for anti-SARS-CoV-2 IgG antibodies.

\subsection{Statistics}

Clinical characteristics were summarized using descriptive statistics. Categorical variables are reported as numbers and percentages. Quantitative variables are reported as medians and interquartile ranges and presented as medians and min to max in the figures. Outliers in experimental data were detected by the application of ROUT method and then excluded from the analysis. The normality of experimental data was evaluated by the Shapiro-Wilk test. Two groups were compared using the Wilcoxon signed-rank test, Mann-Whitney $U$ test or Fisher`s exact test. Three or more groups were compared using the Kruskall-Wallis test followed by Dunn's multiple comparison test or Chi-square test followed by Bonferroni test (the exact method used is stated in the figure legends). Significance between 2 continuous variables was calculated by using a Spearman correlation test. The sample size was not calculated by statistical methods, but was determined based on our previous experience. Statistical significances are indicated in the figures by asterisks as follows ${ }^{*} p<0.05$, ${ }^{* *} p<0.01,{ }^{* * *} p<0.001$ or $^{* * * *} p<0.0001$. Analysis and visualizations were performed using GraphPad Prism.v.8 (GraphPad Software, CA, USA) and SPSS software v.19.0 (SPSS Corp., Armonk, NY, USA).

\subsection{Role of the funding source}

The funders had no role in study design, data collection, analysis and interpretation, writing and submission of the manuscript.

\section{Results}

\subsection{Study cohorts}

We recruited a total of 203 girls, boys and adolescents admitted to different hospitals from Buenos Aires city and surroundings with clinical manifestations of SARS-CoV-2 infection between May and October 2020. Samples from children with COVID-19 and MIS-C were obtained within $24-72 \mathrm{~h}$ of being admitted, after the initiation of therapeutic interventions including steroids. Children with COVID-19 $(n=174)$ included asymptomatic $(n=29)$, mild $(n=102)$ and moderate $(n=43)$ patients. The median age (25th-75th percentile) of COVID-19 group was 4 years $(0.5-10$ years), of whom $45 \%(n=79)$ were girls. Only $1 \%$ $(n=2)$ of children with COVID-19 were admitted to PICU. Table 1 and Table S1 summarize the characteristics of children with COVID-19 included in the study. Regarding the MIS-C group $(n=21)$, the median (25th-75th percentile) age was 6 years (3-10 years), of whom $24 \%$ $(n=5)$ were girls. More than half of MIS-C patients required intensive 
care, 95\% ( $n=20)$ needed steroids and gammaglobulin, 43\% ( $n=9)$ needed inotrope treatment and $24 \%(n=5)$ required mechanical ventilation. Data from MIS-C are shown in Tables 1 and Table S2. Samples from eight children recovered from COVID-19 were obtained within 4 to 5 weeks after the onset of disease. Six of them had mild symptoms and 2 moderate diseases at diagnosis. The median age (25th-75th percentile) of recovered COVID-19 group was 4 years ( $0.6-12$ years), of whom $38 \%$ $(n=3)$ were girls. All recovered children tested positive for SARS-CoV-2 in nasopharyngeal swabs by PCR at hospital admission and $88 \%(n=7)$ were positive for $\operatorname{IgG}$ antibodies directed to SARS-CoV-2. Data of this cohort is shown in Table S3. No fatal outcome was observed in any cohort.

\subsection{Neutrophils from children with symptomatic COVID-19 express an atypical phenotype}

We characterized the phenotype of neutrophils from children with COVID-19 by flow cytometry in whole blood samples. The gating strategy used is shown in Fig. S1. Neutrophil activation is characterized by an increased expression of CD11b and CD66b and a down regulation of L-selectin expression [14,15]. The analysis of neutrophil phenotype in children with COVID-19 revealed a particular signature. In comparison with healthy controls, neutrophils from children with COVID-19 showed a reduced expression of CD11b, CD66b, and Lselectin and an enhanced expression of two inflammatory markers [16-18], HLA-DR and PECAM-1 (Fig. 1a-c). A positive correlation was found between the expression of $\mathrm{CD} 11 \mathrm{~b}$ and $\mathrm{CD} 66 \mathrm{~b}$ in neutrophils from children with COVID-19 (Fig. 1d).
Neutrophil activation is usually associated with an augmented expression of inhibitory receptors that limits its inflammatory potential by increasing the neutrophil activation threshold [19]. As shown in Fig. 2a,b, the expression of the inhibitory receptors LAIR-1 and PDL1 was significantly higher in COVID-19 neutrophils compared with controls. Moreover, considering the important role that the receptors for the Fc portion of IgG ( $\mathrm{Fc} \gamma \mathrm{Rs})$ play in both, the protection against viral infections and the immunopathogenesis associated to viral infections in the airway [20], we also analyzed the expression of Fc $\gamma$ RIII (CD16), Fc $\gamma$ RII (CD32), and Fc $\gamma$ RI (CD64). Neutrophils from children with COVID-19 showed a slight but significant reduction in the expression of CD16 compared with healthy neutrophils, while no changes in the expression of CD32 was observed. Interestingly, whereas no expression of CD64 was found in neutrophils from healthy controls, neutrophils from children with COVID-19 showed a wide range of CD64 expression ranged from 0 to $100 \%$ (Fig. 2c-d). We conclude that neutrophils from children with COVID-19 express a distinct inflammatory phenotype characterized by the down-regulation of cell-surface molecules involved in cell migration and an increased expression of both, inflammatory markers (HLA-DR, PECAM-1 and CD64) and inhibitory molecules (LAIR-1 and PD-L1).

\subsection{Neutrophils from children with COVID-19 and healthy donors show a similar pattern of cytokine production and NETs release}

Previous studies have shown that moderate and severe COVID-19 in adults is associated with increased plasma levels of inflammatory cytokines and NETs [21-23]. As shown in Fig. 3a, the plasma a

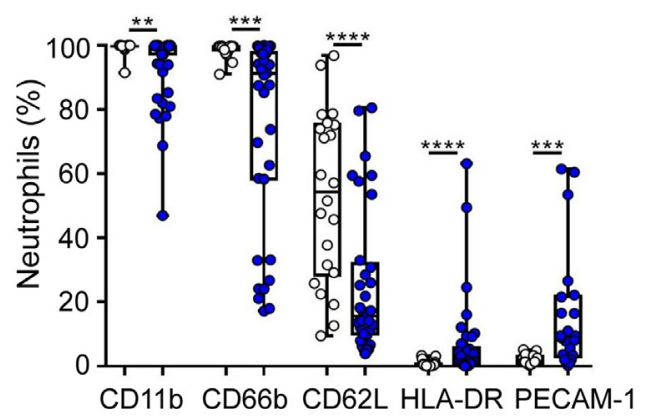

b

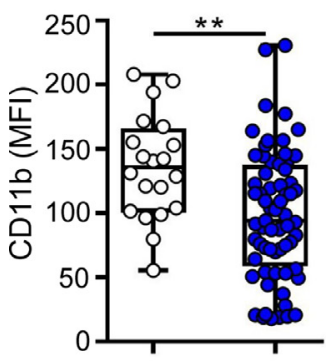

C

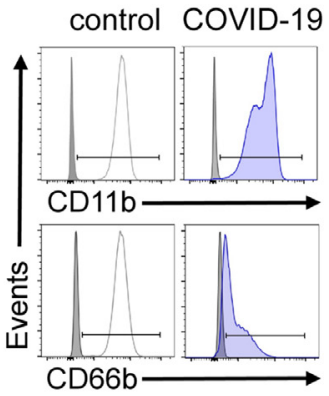

d

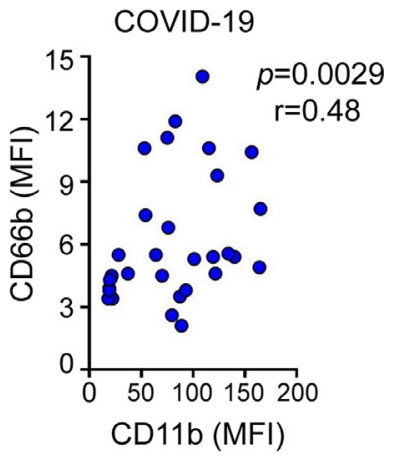

○ control • COVID-19

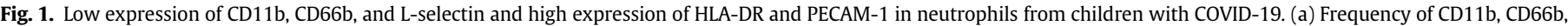

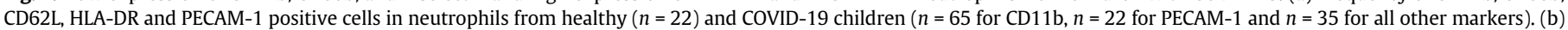

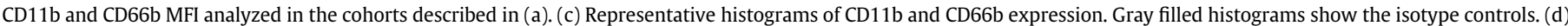

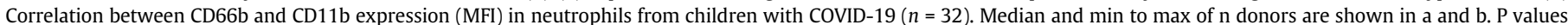

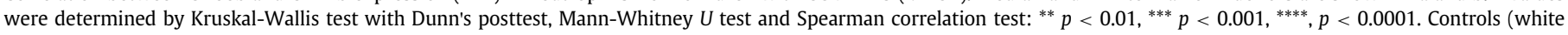
circles), COVID-19 (blue circles) (For interpretation of the references to color in this figure legend, the reader is referred to the web version of this article.). 
a

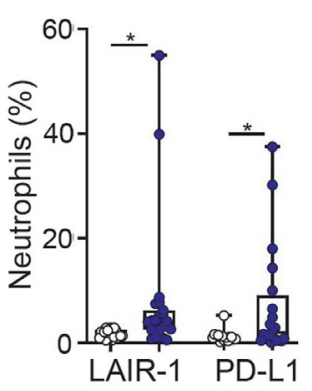

C

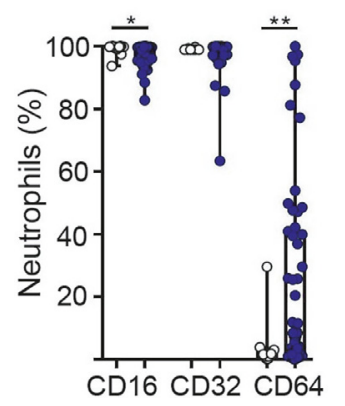

b

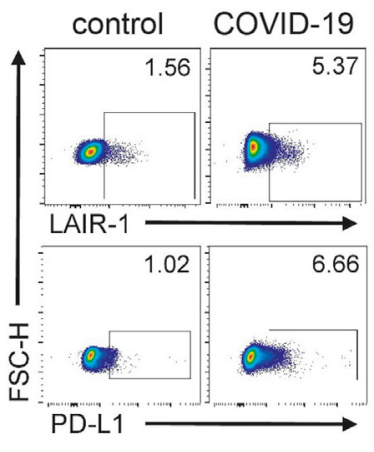

Fig. 2. High expression of LAIR-1, PD-L1 and CD64 in neutrophils from children with COVID-19. (a) Frequency of LAIR-1 and PD-L1 positive cells in neutrophils from healthy $(n=20)$ and COVID-19 $(n=21)$. (b) Representative dot plots of LAIR-1 and PD-L1 expression in a donor from each cohort are shown. Numbers indicate the percentage of neutrophils positive for the expression of LAIR-1 or PD-L1. (c) Frequency of positive cells for CD16, CD32 and CD64 expression in neutrophils from healthy $(n=22)$ and COVID-19 $(n=56)$. (d) Representative dot plots of CD16 and CD64 expression are shown. Data show the percentages of positive cells in gated neutrophils. Median and min to max of $\mathrm{n}$ donors are shown in a and c. $P$ values were determined by KruskalWallis test with Dunn's posttest and Mann-Whitney $U$ test: ${ }^{*} p<0.05$, ${ }^{* *} p<0.01$. Controls (white circle), COVID-19 (blue circle) (For interpretation of the references to color in this figure legend, the reader is referred to the web version of this article.).

concentrations of IL- $1 \beta$, IL- 6 and IL- 8 were below the detection limit of the assay in most of children with COVID-19. In addition, plasma concentrations of both, cell-free DNA and citrullinated histone $\mathrm{H3}$ were similar in COVID-19 and healthy children (Fig. 3b). We then analyzed whether the changes in the phenotype of COVID-19 neutrophils were associated to a higher ability to produce inflammatory cytokines. Purified neutrophils were cultured for $18 \mathrm{~h}$ and the spontaneous production of cytokines was analyzed. Moreover, considering that TLR7 and 8 appear to be involved in the recognition of SARS-CoV-2 genome as well as in the inflammatory responses triggered by SARS-CoV-2 infection [24], we also examined the production of cytokines by neutrophils upon stimulation with the TLR7/8 agonist R848. Neutrophils from healthy donors and children with COVID-19 produced similar levels of cytokines both spontaneously and after being activated by the compound R848 (Fig. 3c-d). Comparable levels of cell-free DNA were also observed in neutrophil supernatants after $18 \mathrm{~h}$ of culture (Fig. 3e).

\subsection{Expression of neutrophil adhesion molecules, inhibitory receptors and production of inflammatory mediators does not distinguish asymptomatic from symptomatic COVID-19 in children}

We analyzed whether there was any association between the changes in neutrophil phenotype or function and disease severity in children with COVID-19. A cohort of MIS-C was also included in the analysis. As shown in Fig. $4 a-d$, the expression of CD11b, CD66b, LAIR-1, and PD-L1 in neutrophils did not distinguish between asymptomatic and symptomatic children with COVID-19. However, all these markers showed a greater expression in neutrophils from MIS-C. Considering that neutrophil expression of CD11b and CD66b among children with COVID-19 shows a wide distribution of values (see Fig. 1a and b), we analyzed whether this heterogeneity could be related to differences in the age or sex of patients. No differences were found when data were stratified according to age, while a higher expression of CD66b was observed in boys compared with girls (Fig. S2a-d).

We then studied whether there was any link between the levels of IL- $1 \beta$ and IL- 8 cytokines produced by neutrophils and disease severity. No significant differences were observed (Fig. 4e). Similarly, the levels of cell-free DNA in both, neutrophil supernatants and plasma, as well as the levels of citrullinated histone $\mathrm{H} 3$ in plasma could not distinguish disease severity in children with COVID-19. However, these parameters were shown to be significantly higher in MIS-C (Fig. 4f-g).

\subsection{Neutrophil expression of CD64 and plasma levels of IgG antibodies directed to the spike protein of SARS-CoV-2 distinguish asymptomatic from symptomatic COVID-19 in children}

Interestingly, we found that the expression of CD64 clearly distinguished asymptomatic from mild and moderate COVID-19. Moreover, the analysis performed in a small cohort of children recently recovered from COVID-19, revealed that the expression of CD64 returned to baseline after recovery (Fig. 5a). No differences in CD64 expression were observed according to the age and sex within the COVID-19 and MIS-C cohorts. (Fig. S3ab).

Severe disease in adult COVID-19 is clearly associated with the presence of high levels of plasma anti-SARS-CoV-2 IgG antibodies [25]. We then examined whether an enhanced antibody response was also linked to disease progression in pediatric COVID-19. The presence of IgG antibodies in plasma was analyzed using an ELISA that detects IgG antibodies directed to the spike protein of SARSCoV-2 [13]. As shown in Fig. 5b, children with COVID-19 showed a marked diversity in the antibody response against SARS-CoV-2, while MIS-C showed a more robust response, perhaps reflecting the time elapsed since the onset of SARS-CoV-2 infection. In fact, more than $90 \%$ of MIS-C had laboratory evidence of previous SARS-CoV-2 exposure. Interestingly, the antibody response against SARS-CoV-2 distinguished asymptomatic from mild and moderate COVID-19 in children. Consistent with the observations made in adult COVID-19 patients [25,26], plasma levels of anti-SARS-CoV-2 IgG antibodies were significantly increased in symptomatic children with COVID-19 (Fig. 5c). As expected, infants under 1 year with COVID-19 had the lowest levels of anti-SARS-CoV-2 IgG antibodies (Fig. S3c). No differences were observed according to the sex within the COVID-19 or MIS-C cohorts. (Fig. S3d).

\section{Discussion}

Very little is known about the immune response against SARSCoV-2 infection in children. Perhaps reflecting that the course of pediatric disease is usually mild, studies have mainly focused on both adults and children with MIS-C. A comprehensive review on the course of infection diseases in children shows that the mild evolution of pediatric COVID-19 does not represent an exception. Indeed, for a variety of infections school-age children have the least severe disease [27]. However, this is not a general rule. Infants and children with secondary heterologous dengue infections are at most risk for severe dengue disease [28]. Moreover, severe respiratory syncytial virus infection is the leading cause of bronchiolitis and hospitalization in infants causing only minor symptoms in adults [29].

Severe COVID-19 in adults is associated with an overactive inflammatory response. It is well established that macrophages and 
a
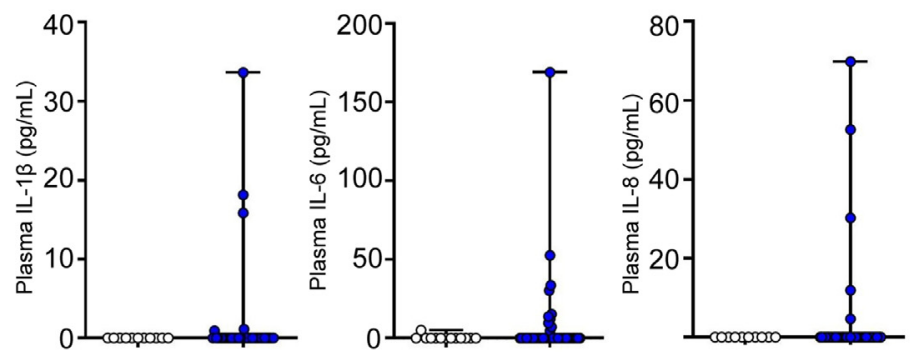

d

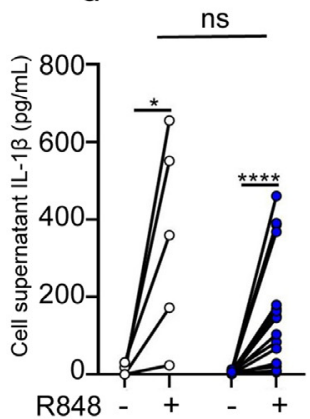

b
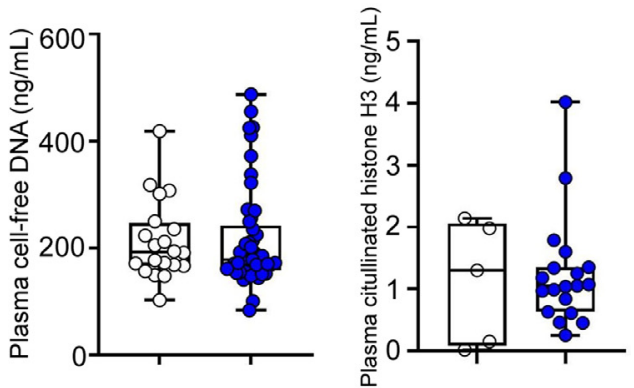

e

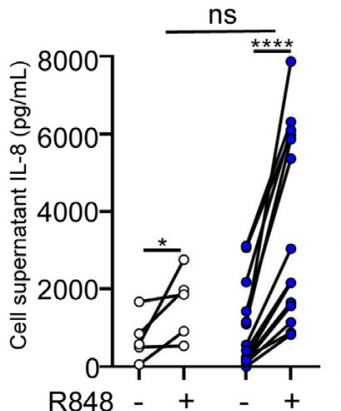

- control - COVID-19

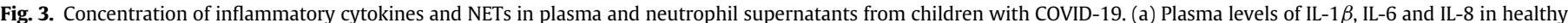

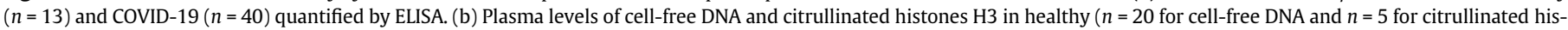

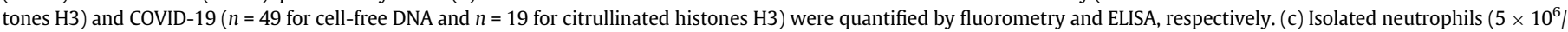

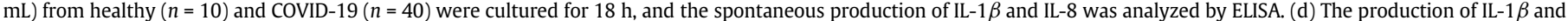

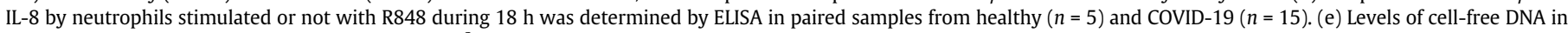

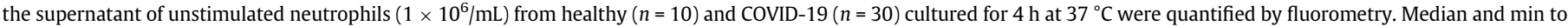

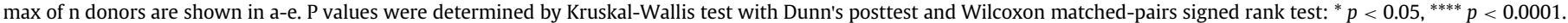

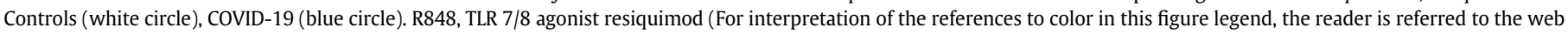
version of this article.).

monocytes are major drivers of this systemic response [30-32], but several lines of evidence suggest that neutrophils also play an important role. Increased neutrophil counts and neutrophil-to-lymphocyte ratio are early indicators of SARS-CoV-2 infection, predicting a worse outcome [33,34]. High number of neutrophils have been found in the bronchoalveolar lavage fluid of severe COVID-19 patients while lung autopsies revealed variable levels of neutrophil infiltration $[21,35,36]$. Moreover, increased blood concentrations of a variety of neutrophil products such as NETs, myeloperoxidase, and calprotectin have been described in patients with severe COVID-19 [22,37,38].

To our knowledge, no previous study has analyzed the properties of neutrophils in pediatric COVID-19. Contrasting with the observations made in adult COVID-19 patients, pediatric COVID-19 is not associated to lymphopenia, neutrophilia, a high neutrophil/lymphocyte ratio or a pro-NETosis neutrophil phenotype. Of note, we found that the phenotype of neutrophils from children with COVID-19 express a particular signature characterized by a down-regulation in the expression of adhesion molecules involved in cell migration together with a high expression of both, the activation markers CD64, HLA-DR and PECAM-1 and the inhibitory receptors LAIR-1 and PD-L1. This phenotype is unusual as neutrophil activation is associated with the up-regulation of adhesion molecules [14] as it was observed in adult COVID-19 [39]. We hypothesized that the downregulation in the expression of adhesion molecules together with a high expression of inhibitory receptors might prevent neutrophil infiltration in the pulmonary capillaries thus providing protection against tissue injury in the children. It is well known that CD11b, CD66b and L-selectin play a critical role in neutrophil migration and lung infiltration during respiratory infections [40,41]. Moreover, recent studies have shown that the inhibitory receptor LAIR-1 prevents neutrophil migration and neutrophil-driven airway inflammation in the course of lung diseases while PD-L1 inhibits the influx of neutrophils to the airway during tuberculosis infection $[42,43]$.

Although children infected by SARS-CoV-2 are less likely to course severe illness compared with adults, they are still at risk of developing a number of complications. In fact, the clinical spectrum of pediatric COVID-19 includes asymptomatic as well as patients with mild, moderate, severe and critical disease. Our patient cohort includes 174 patients with COVID-19 of which $17 \%$ were asymptomatic, and $59 \%$ and $25 \%$ had a mild and moderate disease, respectively. Our observations reveal that either the neutrophil expression of CD64 or the plasma levels of IgG antibodies directed to the spike protein of SARS-CoV-2 are valuable biomarkers to distinguish asymptomatic from symptomatic children with COVID-19. These parameters have previously associated with severe disease in adult COVID-19, and might be used to stratify patients at admission and identify patients potentially at risk [44].

The type I IgG receptor ( $\mathrm{Fc} \gamma \mathrm{RI}$ or $\mathrm{CD} 64)$ is the only $\mathrm{Fc} \gamma \mathrm{R}$ that binds monomeric IgG with high affinity. Many studies have shown that CD64 is an early and predictive biomarker of neonatal and adult sepsis $[45,46]$. It is normally expressed on monocytes and macrophages but it is rapidly up-regulated in neutrophils in the course of severe inflammatory processes [47]. CD64 expression has been recently reported as a neutrophil activation marker in MIS-C. In the acute phase of MIS-C the neutrophil expression of CD64 is notably increased compared with healthy donors but normalized by the 
a

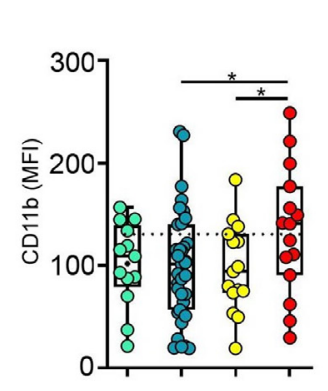

b

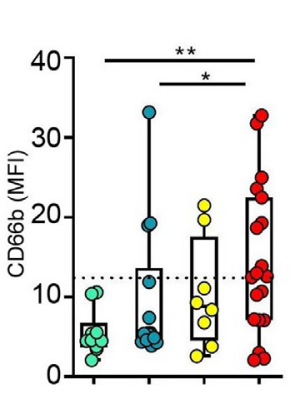

C

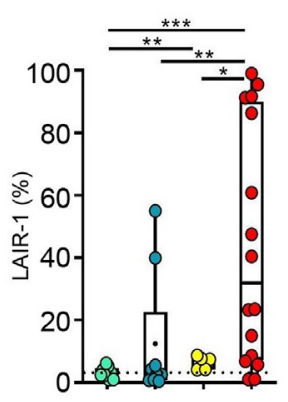

d

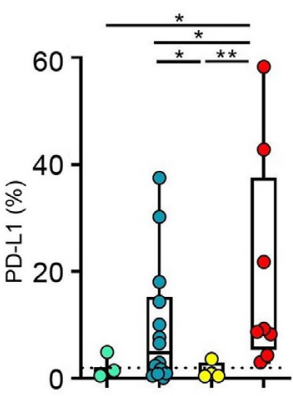

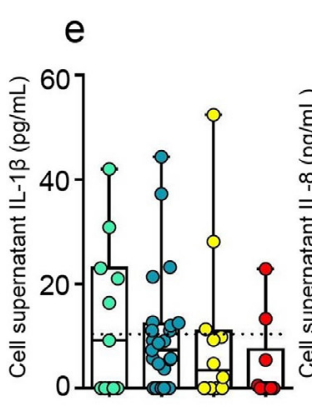
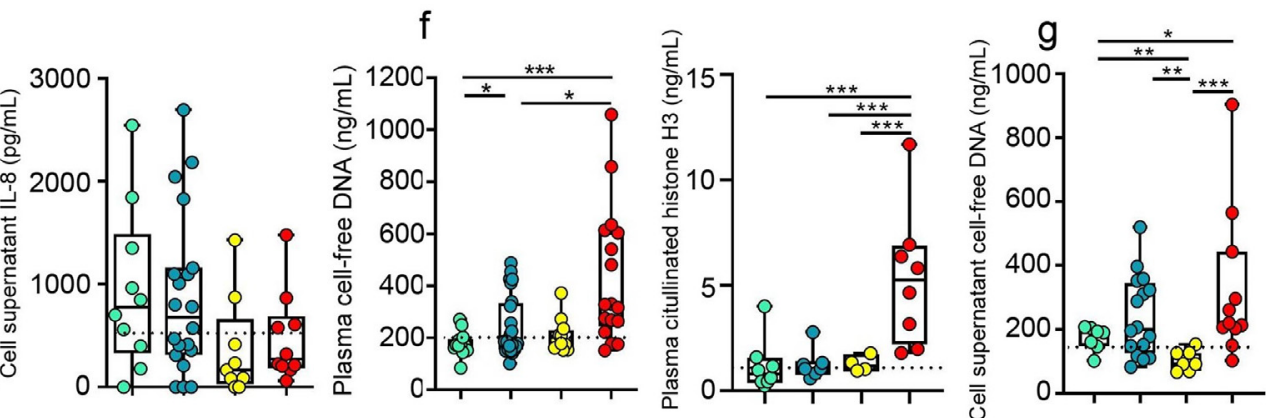

$\frac{\text { o asymptomatic o mild o moderate }}{\text { COVID-19 }}$

- MIS-C

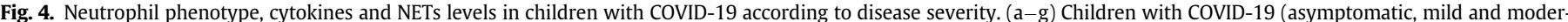

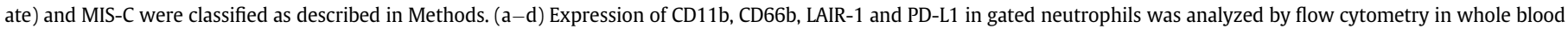

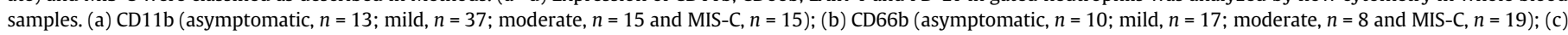

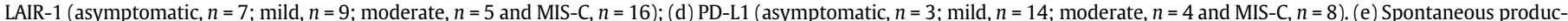

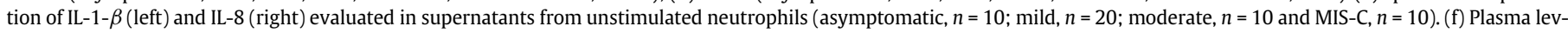

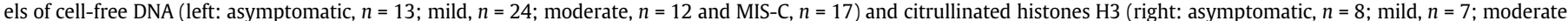

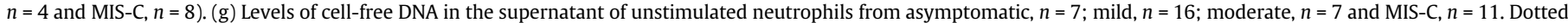

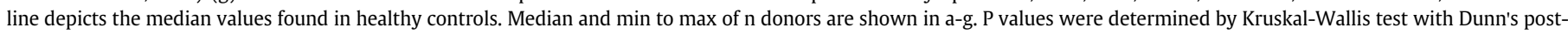

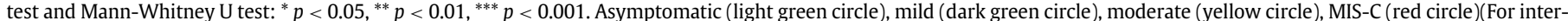
pretation of the references to color in this figure legend, the reader is referred to the web version of this article.).
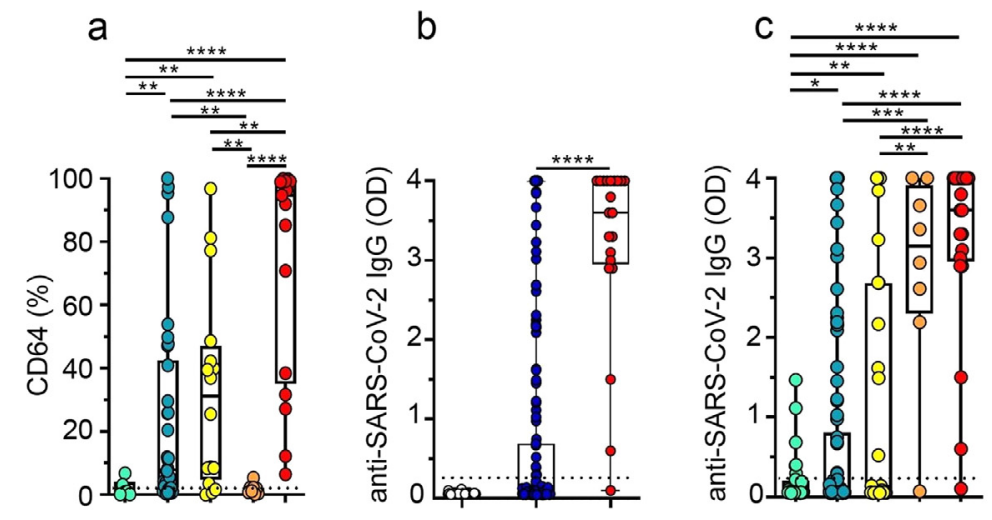

$$
\text { - control • COVID-19 oasymptomatic o mild omoderate o recovered - MIS-C }
$$

\section{COVID-19}

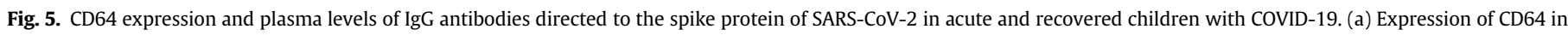

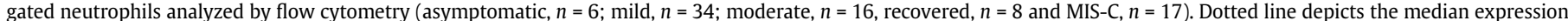

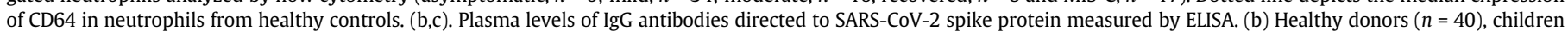

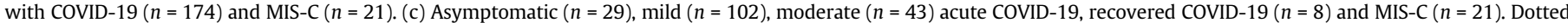

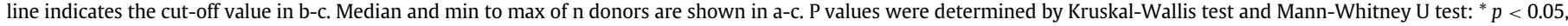

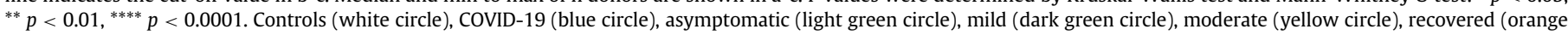
circle), MIS-C (red circle)(For interpretation of the references to color in this figure legend, the reader is referred to the web version of this article.). 
resolution phase [44]. In this sense, we wonder if a persistent expression of CD64 after resolution of acute COVID-19 in children might be a marker capable to predict a worse outcome and progression to MIS-C.

As stated before, asymptomatic and symptomatic children with COVID-19 could be also discriminated by plasma levels of IgG antibodies directed to the spike protein of SARS-CoV-2. This finding is consistent with a large body of evidence in adults with COVID-19 indicating that disease severity is associated with a greater antibody response $[48,49]$. We also observed that MIS-C cohort shows a more robust antibody response compared with children with COVID-19. Considering that MIS-C is associated with a prior exposure to SARS$\mathrm{CoV}-2$, this observation might just mirror the differential time elapsed since the onset of infection between both group of patients. Indeed, Farber and coworkers have recently shown that children with either acute-COVID-19 (16 days post-symptoms onset) or MIS-C develop a similar antibody response characterized by a reduced neutralizing activity compared with adult COVID-19, suggesting a less protective serological response [25].

This study has some limitations. It is important to emphasize that our cohort did not include any children under severe COVID19. Thus, our findings do not differentiate between all clinical spectrum of COVID-19 and MIS-C. Indeed, biomarkers such as neutrophil expression of CD64 and inhibitory receptors and plasma levels of NETs distinguished among asymptomatic, mild or moderate COVID-19 and MIS-C. However, these markers may not be able to distinguish severe COVID-19 from MIS-C, two clinical conditions that might share some pathogenic mechanisms. This issue should be addressed in future cohorts. A second limitation is related to the fact that almost all blood samples from children with MIS-C were obtained after starting therapeutic interventions. Moreover, we were unable to obtain paired samples from infected children during the acute and convalescent period. On the other hand, the small volume of blood collected from each patient limited the performance of studies aimed at characterizing the functionality of neutrophils. Finally, chest CT scans were not carried out except for a small group of patients. Therefore, we could not analyze a possible correlation between neutrophil phenotype and the degree of lung infiltration.

We still do not understand the reasons why children manage to face the challenge of SARS-CoV-2 infection so efficiently. As mentioned above, the control of infection does not appear to rely on a more robust antibody response. The function of $\mathrm{T}$ cells in pediatric COVID-19 remains unexplored. Perhaps the basis of the effective control of SARS-CoV-2 infection in children resides, at least in part, in the innate immune response. Our present observations suggest that infection by SARS-CoV-2 in children is associated to the acquisition of an unconventional phenotype by neutrophils that might prevent lung infiltration preserving tissue integrity.

\section{Declaration of Competing Interest}

The authors have declared that no conflict of interest exists.

\section{Author contributions}

V.S., I.S., M.P.H. and L.A performed experiments and analyzed data. S.C.R., S.C.A., N.A.G., D.F., N.D.C., S.D.L., H.C., M.J.C., C.N.M, L.I.G., G.G., M.S., A.L.A., C.D., M.J.B., N.S., M.F.P., V.N., C.B., P.T., M.T.A., H.P., S.V.N., P.S., E.M.T., M.G., J.L., M.J.R. and P.C. coordinated patient enrollment and sample acquisition and ethical approval of the study. V.S., S.C.R., J.G. and L.A. conceived the study, coordinated experiments, conducted data analyses and draft the manuscript. All authors provided scientific input, read, and approved the manuscript.

\section{Data sharing statement}

Any data not published within the article will be shared in anonymized format by request from any qualified investigator. If desired, please contact the corresponding author of this article.

\section{Acknowledgments}

We express our sincerest thanks to the physicians, nurses, respiratory therapists, and advanced practice providers who cared for these patients for their contribution. Most of all, we are indebted to all the participating children and their families. See Supplemental Acknowledgments for consortium details.

This work was supported by grants from the National Agency for Promotion of Science and Technology, Argentina (IP-COVID-19-0277 and PMO BID PICT 2018-2548 to L.A.) and University of Buenos Aires (20020170100573BA to J.G).

\section{Supplementary materials}

Supplementary material associated with this article can be found in the online version at doi:10.1016/j.ebiom.2021.103357.

\section{References}

[1] Castagnoli R, Votto M, Licari A, Brambilla I, Bruno R, Perlini S, et al. Severe acute respiratory syndrome coronavirus 2 (SARS-CoV-2) infection in children and adolescents: a systematic review. JAMA Pediatr 2020;174(9):882-9.

[2] Lu X, Zhang L, Du H, Zhang J, Li YY, Qu J, et al. SARS-CoV-2 infection in children. N Engl J Med 2020;382(17):1663-5

[3] Diorio C, Henrickson SE, Vella LA, McNerney KO, Chase J, Burudpakdee C, et al. Multisystem inflammatory syndrome in children and COVID-19 are distinct presentations of SARS-CoV-2. J Clin Invest 2020;130(11):5967-75.

[4] Bunyavanich S, Do A, Vicencio A. Nasal gene expression of angiotensin-converting enzyme 2 in children and adults. JAMA 2020;323(23):2427-9.

[5] Ortiz ME, Thurman A, Pezzulo AA, Leidinger MR, Klesney-Tait JA, Karp PH, et al. Heterogeneous expression of the SARS-coronavirus-2 receptor ACE2 in the human respiratory tract. EBioMedicine 2020;60:102976.

[6] Sajuthi SP, DeFord P, Li Y, Jackson ND, Montgomery MT, Everman JL, et al. Type 2 and interferon inflammation regulate SARS-CoV-2 entry factor expression in the airway epithelium. Nat Commun 2020;11(1):5139.

[7] Lingappan K, Karmouty-Quintana H, Davies J, Akkanti B, Harting MT. Understanding the age divide in COVID-19: why are children overwhelmingly spared? Am J Physiol Lung Cell Mol Physiol 2020;319(1):L39-44.

[8] Tosif S, Neeland MR, Sutton P, Licciardi PV, Sarkar S, Selva KJ, et al. Immune responses to SARS-CoV-2 in three children of parents with symptomatic COVID19. Nat Commun 2020;11(1):5703.

[9] Hue S, Beldi-Ferchiou A, Bendib I, Surenaud M, Fourati S, Frapard T, et al. Uncontrolled innate and impaired adaptive immune responses in patients with COVID19 acute respiratory distress syndrome. Am J Respir Crit Care Med 2020;202 (11):1509-19.

[10] Boyum A. Isolation of mononuclear cells and granulocytes from human blood. isolation of monuclear cells by one centrifugation, and of granulocytes by combining centrifugation and sedimentation at 1g. Scand J Clin Lab Investig Suppl 1968;97:77-89.

[11] Schreiber A, Rousselle A, Becker JU, von Massenhausen A, Linkermann A, Kettritz R. Necroptosis controls NET generation and mediates complement activation, endothelial damage, and autoimmune vasculitis. Proc Natl Acad Sci USA 2017:114(45):E9618, -E25

[12] Ramos MV, Mejias MP, Sabbione F, Fernandez-Brando RJ, Santiago AP, Amaral $\mathrm{MM}$, et al. Induction of neutrophil extracellular traps in shiga toxin-associated hemolytic uremic syndrome. J Innate Immun 2016;8(4):400-11.

[13] Ojeda DS, Gonzalez Lopez Ledesma MM, Pallares HM, Costa Navarro GS, Sanchez L, Perazzi B, et al. Emergency response for evaluating SARS-CoV-2 immune status, seroprevalence and convalescent plasma in Argentina. PLoS Pathog 2021;17(1): e1009161.

[14] Silvestre-Roig C, Fridlender ZG, Glogauer M, Scapini P. Neutrophil diversity in health and disease. Trends Immunol 2019;40(7):565-83

[15] Pillay J, Kamp VM, van Hoffen E, Visser T, Tak T, Lammers JW, et al. A subset of neutrophils in human systemic inflammation inhibits $T$ cell responses through mac-1. J Clin Invest 2012;122(1):327-36.

[16] Vono M, Lin A, Norrby-Teglund A, Koup RA, Liang F, Lore K. Neutrophils acquire the capacity for antigen presentation to memory $\mathrm{CD}_{4}{ }^{+} T$ cells in vitro and ex vivo. Blood 2017;129(14):1991-2001

[17] Dangerfield J, Larbi KY, Huang MT, Dewar A, Nourshargh S. PECAM-1 (CD31) homophilic interaction up-regulates alpha6beta1 on transmigrated neutrophils in vivo and plays a functional role in the ability of alpha6 integrins to mediate 
leukocyte migration through the perivascular basement membrane. J Exp Med 2002;196(9):1201-11

[18] Davis RE, Sharma S, Conceicao J, Carneiro P, Novais F, Scott P, et al. Phenotypic and functional characteristics of HLA-DR(+) neutrophils in Brazilians with cutaneous leishmaniasis. J Leukoc Biol 2017;101(3):739-49.

[19] Favier B. Regulation of neutrophil functions through inhibitory receptors: an emerging paradigm in health and disease. Immunol. Rev 2016;273(1):140-55.

[20] Bournazos S, Gupta A, Ravetch JV. The role of IgG Fc receptors in antibody-dependent enhancement. Nature reviews Immunology 2020;20(10):633-43.

[21] Leppkes M, Knopf J, Naschberger E, Lindemann A, Singh J, Herrmann I, et al. Vascular occlusion by neutrophil extracellular traps in COVID-19. EBioMedicine 2020;58:102925.

[22] Zuo Y, Yalavarthi S, Shi H, Gockman K, Zuo M, Madison JA, et al. Neutrophil extracellular traps in COVID-19. JCI insight 2020;5(11).

[23] McElvaney OJ, McEvoy NL, McElvaney OF, Carroll TP, Murphy MP, Dunlea DM, et al. Characterization of the inflammatory response to severe COVID-19 Illness. Am J Respir Crit Care Med 2020;202(6):812-21.

[24] Khanmohammadi S, Rezaei N. Role of toll-like receptors in the pathogenesis of COVID-19. J Med Virol 2021.

[25] Weisberg SP, Connors TJ, Zhu Y, Baldwin MR, Lin WH, Wontakal S, et al. Distinct antibody responses to SARS-CoV-2 in children and adults across the COVID-19 clinical spectrum. Nat Immunol 2021;22(1):25-31.

[26] Liu L, To KK, Chan KH, Wong YC, Zhou R, Kwan KY, et al. High neutralizing antibody titer in intensive care unit patients with COVID-19. Emerg Microbes Infect 2020;9(1):1664-70

[27] Glynn JR, Moss PAH. Systematic analysis of infectious disease outcomes by age shows lowest severity in school-age children. Sci Data 2020;7(1):329.

[28] Burattini MN, Lopez LF, Coutinho FA, Siqueira Jr. JB, Homsani S, Sarti E, et al. Age and regional differences in clinical presentation and risk of hospitalization for dengue in Brazil, 2000-2014. Clinics 2016;71(8):455-63.

[29] Geoghegan S, Erviti A, Caballero MT, Vallone F, Zanone SM, Losada JV, et al. Mortality due to respiratory syncytial virus. burden and risk factors. Am J Respir Crit. Care Med 2017;195(1):96-103.

[30] Merad M, Martin JC. Pathological inflammation in patients with COVID-19: a key role for monocytes and macrophages. Nat Rev Immunol 2020;20(6):355-62.

[31] Sanchez-Cerrillo I, Landete P, Aldave B, Sanchez-Alonso S, Sanchez-Azofra A, Marcos-Jimenez A, et al. COVID-19 severity associates with pulmonary redistribution of CD1c+ DCs and inflammatory transitional and nonclassical monocytes. J Clin Invest 2020;130(12):6290-300.

[32] Wang C, Xie J, Zhao L, Fei X, Zhang H, Tan Y, et al. Alveolar macrophage dysfunction and cytokine storm in the pathogenesis of two severe COVID-19 patients. EBioMedicine 2020;57:102833.

[33] Parackova Z, Zentsova I, Bloomfield M, Vrabcova P, Smetanova J, Klocperk A, et al. Disharmonic inflammatory signatures in COVID-19: augmented neutrophils' but impaired monocytes' and dendritic cells responsiveness. Cells 2020;9(10).

[34] Chen G, Wu D, Guo W, Cao Y, Huang D, Wang H, et al. Clinical and immunological features of severe and moderate coronavirus disease 2019. J Clin Invest 2020;130 (5):2620-9.
[35] Barnes BJ, Adrover JM, Baxter-Stoltzfus A, Borczuk A, Cools-Lartigue J, Crawford JM, et al. Targeting potential drivers of COVID-19: neutrophil extracellular traps. J Exp Med 2020;217(6).

[36] Zhou Z, Ren L, Zhang L, Zhong J, Xiao Y, Jia Z, et al. Heightened innate immune responses in the respiratory tract of COVID-19 patients. Cell Host Microbe 2020;27(6):883-890.e2.

[37] Silvin A, Chapuis N, Dunsmore G, Goubet AG, Dubuisson A, Derosa L, et al. Elevated calprotectin and abnormal myeloid cell subsets discriminate severe from mild COVID-19. Cell 2020;182(6) 1401-18 e18

[38] Wu C, Chen X, Cai Y, Xia J, Zhou X, Xu S, et al. Risk factors associated with acute respiratory distress syndrome and death in patients with coronavirus disease 2019 pneumonia in Wuhan, China. JAMA Intern Med 2020;180(7):934-43.

[39] Carissimo G, Xu W, Kwok I, Abdad MY, Chan YH, Fong SW, et al. Whole blood immunophenotyping uncovers immature neutrophil-to-VD2 T-cell ratio as an early marker for severe COVID-19. Nat Commun 2020;11(1):5243.

[40] Duan M, Steinfort DP, Smallwood D, Hew M, Chen W, Ernst M, et al. CD11b immunophenotyping identifies inflammatory profiles in the mouse and human lungs. Mucosal Immunol 2016;9(2):550-63.

[41] Scott NR, Swanson RV, Al-Hammadi N, Domingo-Gonzalez R, Rangel-Moreno J Kriel BA, et al. S100A8/A9 regulates CD11b expression and neutrophil recruitment during chronic tuberculosis. J. Clin. Invest. 2020;130(6):3098-112.

[42] Kumawat K, Geerdink RJ, Hennus MP, Roda MA, van Ark I, Leusink-Muis T, et al. LAIR-1 limits neutrophilic airway inflammation. Front Immunol 2019;10:842.

[43] Lazar-Molnar E, Chen B, Sweeney KA, Wang EJ, Liu W, Lin J, et al. Programmed death-1 (PD-1)-deficient mice are extraordinarily sensitive to tuberculosis. Proc Natl Acad Sci USA 2010;107(30):13402-7.

[44] Carter MJ, Fish M, Jennings A, Doores KJ, Wellman P, Seow J, et al. Periphera immunophenotypes in children with multisystem inflammatory syndrome associated with SARS-CoV-2 infection. Nat Med 2020;26(11):1701-7.

[45] Dimoula A, Pradier O, Kassengera Z, Dalcomune D, Turkan H, Vincent JL. Serial determinations of neutrophil CD64 expression for the diagnosis and monitoring of sepsis in critically ill patients. Clin Infect Dis Off Publ Infect Dis Soc Am 2014;58 (6):820-9.

[46] Nuutila J. The novel applications of the quantitative analysis of neutrophil cel surface fcgammaRI (CD64) to the diagnosis of infectious and inflammatory diseases. Curr Opin Infect Dis 2010;23(3):268-74.

[47] Schiff DE, Rae J, Martin TR, Davis BH, Curnutte JT. Increased phagocyte Fc gammaRI expression and improved Fc gamma-receptor-mediated phagocytosis after in vivo recombinant human interferon-gamma treatment of normal human subjects. Blood 1997:90(8):3187-94.

[48] Gong F, Dai Y, Zheng T, Cheng L, Zhao D, Wang H, et al. Peripheral $\mathrm{CD}_{4}+\mathrm{T}$ cell subsets and antibody response in COVID-19 convalescent individuals. J Clin Invest 2020;130(12):6588-99.

[49] Lucas C, Klein J, Sundaram M, Liu F, Wong P, Silva J, et al. Kinetics of antibody responses dictate COVID-19 outcome. medRxiv 2020 the preprint server for health sciences. doi: 10.1016/2020.12.18.20248331. 